\title{
Requirement of cholesterol for calcium-dependent vesicle fusion by stabilizing synaptotagmin-1- induced membrane bending
}

Yongsoo Park ( $\nabla$ ypark@hbku.edu.qa )

Qatar Biomedical Research Institute (QBRI), Hamad Bin Khalifa University (HBKU)

Houda Yasmine Ali Moussa

Qatar Biomedical Research Institute (QBRI), Hamad Bin Khalifa University (HBKU)

Kyung Chul Shin

Qatar Biomedical Research Institute (QBRI), Hamad Bin Khalifa University (HBKU)

Janarthanan Ponraj

Qatar Environment and Energy Research Institute (QEERI), Hamad Bin Khalifa University (HBKU)

Said Mansour

Qatar Environment and Energy Research Institute (QEERI), Hamad Bin Khalifa University (HBKU)

Je-Kyung Ryu

TU Delft https://orcid.org/0000-0002-0545-9502

Soo Jin Kim

Pohang University of Science and Technology

Article

Keywords:

Posted Date: October 3rd, 2022

DOI: https://doi.org/10.21203/rs.3.rs-1313659/v2

License: (9) This work is licensed under a Creative Commons Attribution 4.0 International License.

Read Full License 


\section{Abstract}

Cholesterol is essential for neuronal activity and function. Cholesterol depletion in the plasma membrane impairs synaptic transmission. However, the molecular mechanisms by which cholesterol deficiency leads to defects in vesicle fusion remain poorly understood. Here we show that cholesterol is required for $\mathrm{Ca} 2+-$ dependent native vesicle fusion using the in-vitro reconstitution of fusion and amperometry to monitor exocytosis in chromaffin cells. Purified native vesicles were crucial for the reconstitution of physiological Ca2+-dependent fusion, whereas vesicle-mimicking liposomes failed to reproduce the cholesterol effect. Intriguingly, cholesterol had no effect on membrane binding of synaptotagmin-1, a $\mathrm{Ca} 2+$ sensor for ultrafast fusion. Cholesterol stabilizes local membrane bending induced by synaptotagmin-1, thereby lowering the energy barrier for $\mathrm{Ca} 2+$-dependent fusion to occur. Our data provide evidence that cholesterol depletion abolishes $\mathrm{Ca} 2+-$ dependent vesicle fusion by disrupting synaptotagmin-1-induced membrane bending, and suggests that cholesterol is an important lipid regulator for $\mathrm{Ca} 2+-$ dependent fusion.

\section{Background}

Cholesterol is a major component in cell membrane bilayers, and is essential for membrane structure and fluidity. Brain is the most cholesterol-enriched organ and a human brain contains about $20-25 \%$ of the body's cholesterol ${ }^{1,2}$; this high density suggests that cholesterol has a critical function in the brain. Agerelated cholesterol reduction in the frontal and temporal cortices ${ }^{3}$ results in loss of synaptic contacts, changes in neuronal morphology, and reduced synaptic plasticity ${ }^{4}$. Cholesterol is associated with neurogenesis, neurodevelopment, and synaptogenesis ${ }^{5,6}$. Age-related cholesterol deficiency in the plasma membrane leads to deficits in synaptic plasticity in mouse hippocampal neurons ${ }^{7}$, and cholesterol depletion by methyl- $\beta$-cyclodextrin (MCD) from the plasma membrane impairs neurotransmission and neuronal activity, and thereby leads to synapse degeneration ${ }^{8,9}$.

The plasma membrane contains $\sim 80 \%$ of total cellular cholesterol ${ }^{10}$. Cholesterol is capable of clustering syntaxin-1 $\mathrm{A}^{11}$ so that soluble $\mathrm{N}$-ethylmaleimide-sensitive factor attachment protein receptor (SNARE) proteins become concentrated in cholesterol-enriched domains in the plasma membrane ${ }^{12}$. Depletion and reduction of cholesterol level in the plasma membrane inhibit $\mathrm{Ca}^{2+}$-dependent exocytosis of large densecore vesicles (LDCVs) ${ }^{13}$ and cortical secretory vesicles from sea urchins ${ }^{14}$, as well as synaptic vesicles in hippocampal neurons ${ }^{8}, 15$, cortical synaptosomes ${ }^{16}$, ribbon synapses ${ }^{17}$, and motor nerve terminals ${ }^{18}$. However, the molecular mechanisms by which cholesterol deficiency disrupts synaptic transmission and induces neurodegeneration remain elusive and controversial.

Exocytosis is the process of neurotransmitter release through merging two lipid bilayers. It is mediated by SNARE proteins ${ }^{19,20}$. Neuronal SNARE proteins consist of Q-SNARE in the plasma membrane (syntaxin-1 and SNAP-25) and R-SNARE in the vesicle membrane (synaptobrevin-2 or vesicle-associated membrane protein-2 (VAMP-2) $)^{19}$. Synaptotagmin-1 is responsible for ultrafast $\mathrm{Ca}^{2+}$-dependent exocytosis. 
Synaptotagmin-1 contains two tandem C2-domains that coordinates $\mathrm{Ca}^{2+}$ binding; the $\mathrm{Ca}^{2+}$-bound $\mathrm{C} 2$ domain can be inserted to negatively charged anionic phospholipids by electrostatic interaction ${ }^{20}$. In spite of intense investigation of synaptotagmin-1, the molecular mechanisms by which synaptotagmin- 1 mediates $\mathrm{Ca}^{2+}$-dependent vesicle are still under debate ${ }^{21}$.

Here we propose novel mechanisms by which cholesterol in the plasma membrane mediates synaptotagmin-1-induced vesicle fusion. We used amperometry to monitor exocytosis in real time, and performed in-vitro reconstitution of vesicle fusion including large dense-core vesicles (LDCVs) and synaptic vesicles (SVs), and showed that cholesterol is required for $\mathrm{Ca}^{2+}$-dependent vesicle fusion. Importantly, vesicle-mimicking liposomes fail to reproduce the cholesterol effect, but purified native vesicles, i.e., LDCVs and SVs, are crucial for the reconstitution of physiological $\mathrm{Ca}^{2+}$-dependent fusion. Membrane binding of synaptotagmin-1 occurs regardless of cholesterol. Transmission electron microscope (TEM) revealed that cholesterol stabilizes membrane bending and curvature induced by the insertion of synaptotagmin-1, and therefore the membrane bending energy can lower the energy barrier to fusion.

\section{Results}

\section{Cholesterol depletion causes deficits in exocytosis.}

We investigated whether local membrane curvature is observable in-vivo using primary chromaffin cells. Using electron microscopy (Fig. 1a-f) we observed the invagination of the plasma membrane into LDCVs, suggesting that the plasma membrane could be already curved before $\mathrm{Ca}^{2+}$ triggering. Cholesterol induces spontaneous membrane curvature and bending 22,23 , thereby promoting highly curved membrane intermediate structures in membrane fusion ${ }^{24,25}$. Moreover, high-curvature membrane domains such as caveolae are heavily enriched in cholesterol, which is essential for membrane invaginations ${ }^{22,26}$. Cholesterol depletion by methyl- $\beta$-cyclodextrin $(M C D)$ or nystatin disrupts membrane invaginations and leads to a flattening of the curved caveolae membrane structure 27,28 .

Given that the curved and invaginated membranes are enriched with cholesterol $22,27,28$ and $~ 67 \%$ vesicles are proximal to the invaginated plasma membranes (Fig. 1e), the membrane invagination to LDCVs we observed is likely to be cholesterol-enriched regions, where SNARE proteins are present ${ }^{29,30}$ (Fig. 1a-e). To test the cholesterol effect on vesicle fusion, we used amperometry to monitor LDCV exocytosis in real time. Indeed, treatment with MCD, which depletes cholesterol from the plasma membrane, inhibited $\mathrm{Ca}^{2+}$-dependent LDCV fusion by 60\% (Fig. 1g-i).

\section{Without cholesterol, no $\mathrm{Ca}^{2+}$-dependent vesicle fusion.}

To further study the molecular mechanisms by which cholesterol deficiency affects vesicle fusion, we applied a reconstitution system of vesicle fusion by using purified native vesicles such as LDCVs and 
SVs, as reported previously $31,32,33$. The plasma membrane-mimicking liposomes (PM-liposomes) contain the stabilized Q-SNARE complex (syntaxin-1A and SNAP-25A in a 1:1 molar ratio ${ }^{34}$ )(Online Methods).

We first tested the effect of cholesterol on basal fusion without $\mathrm{Ca}^{2+}$. LDCV fusion with the PM-liposomes was readily observed (Fig. 2a), but the absence of cholesterol in the PM-liposomes reduced LDCV fusion by $~ 50 \%$ (Fig. $2 \mathrm{a}$,d); the PM-liposomes contain either $25 \%$ or $0 \%$ cholesterol (Chol). The efficiency of the SNARE assembly and SNARE complex formation was examined using the light chain of tetanus toxin (TeNT), a protease specific for free VAMP-2; i.e. VAMP-2 in the assembled SNARE complexes is resistant to the cleavage by TeNT so that TeNT-resistant VAMP-2 represents the ternary SNARE complex formation ${ }^{31}$. After LDCV fusion with the PM-liposomes as a fusion assay in Fig. 2a, TeNT was added to cleave free VAMP-2 and quantify VAMP-2 engaged in the SNARE complex formation. Absence of cholesterol $(0 \% \mathrm{Chol})$ in liposomes slightly reduced the ternary SNARE complex formation compared to liposomes containing cholesterol $(25 \%$ Chol) (Fig. 2b); this result suggests that cholesterol facilitates basal fusion of LDCVs by increasing the efficiency of SNARE assembly. Cholesterol can enhance SNAREcomplex formation, by inducing clustering of SNARE proteins in the plasma membrane $e^{11,12,30}$.

Then we investigated the cholesterol effect on $\mathrm{Ca}^{2+}$-dependent fusion. Although cholesterol controls $\mathrm{Ca}^{2+}$-dependent neurotransmission and exocytosis in diverse cell types $8,13,14,15,16,17,18$, the step at which vesicle fusion is impaired has not been determined. We used native vesicles to completely reconstitute vesicle fusion that reproduces $\mathrm{Ca}^{2+}$-dependent vesicle fusion, correlating with the in-vivo data in a physiological ionic environment ${ }^{31,32,33}$. Addition of $100 \mu \mathrm{M}$ free $\mathrm{Ca}^{2+}$ accelerated vesicle fusion in the presence of $\mathrm{Mg}^{2+} /$ ATP (Fig. 2c,d), where synaptotagmin-1 interacts only with $\mathrm{PIP}_{2}$-containing membranes, but not SNARE proteins ${ }^{31,32}$. VAMP-2 ${ }_{1-96}$, the soluble cytoplasmic region of VAMP-2, blocked vesicle fusion; this result supports SNARE-dependent vesicle fusion. Surprisingly, $\mathrm{Ca}^{2+}{ }^{2+}$-evoked LDCV fusion was completely abolished when cholesterol in the PM-liposomes was absent (Fig. 2c,d). The Q-SNARE proteins were incorporated in the PM-liposomes independently of cholesterol (Supplementary Fig. 1). We also confirmed that $\mathrm{Ca}^{2+}$-dependent LDCV fusion did not occur without cholesterol $(0 \% \mathrm{Chol})$ in the PM-liposomes when the full-length syntaxin-1A and SNAP-25A binary acceptor complex (see Online Methods) was included (Supplementary Fig. 2). High concentration of free $\mathrm{Ca}^{2+}$ failed to increase vesicle fusion when the PM-liposomes contained no cholesterol (Fig. 2e) and cholesterol accelerated $\mathrm{Ca}^{2+}$ dependent LDCV fusion in a dose-dependent manner (Fig. 2f). Altogether, our results confirm that cholesterol is required for $\mathrm{Ca}^{2+}$-dependent vesicle fusion and that this in-vitro reconstitution reproduces physiological roles of cholesterol on vesicle fusion and exocytosis.

\section{Cholesterol is not required for liposome-liposome fusion.}

We further tested SVs purified from mice brain to determine whether cholesterol is essential for $\mathrm{Ca}^{2+}$ dependent SV fusion. $\mathrm{Ca}^{2+}$ failed to accelerate SV fusion in the absence of cholesterol in the PMliposomes $(0 \% \mathrm{Chol})$ (Fig. 3a-c); this result is consistent with the cholesterol requirement for $\mathrm{Ca}^{2+}-$ 
dependent LDCV fusion (Fig. 2d), however, $\mathrm{Ca}^{2+}{ }^{2+}$ independent basal SV fusion was not affected (Fig. 3c). A unique and major advantage of native vesicles for a fusion assay is that they maintain the native lipid and protein diversity as well as the structural integrity of vesicles to mimic endogenous vesicle fusion. Instead of purified native vesicles, vesicle-mimicking liposomes (V-liposomes) have been used for a fusion assay to study the molecular mechanisms. We therefore tested V-liposomes that incorporate full length VAMP-2 and synaptotagmin-1 to determine whether the dependence on cholesterol for $\mathrm{Ca}^{2+}{ }_{-}$ dependent fusion can be reproduced (Fig. 3d-f). Intriguingly, $\mathrm{Ca}^{2+}$-dependent liposome fusion was slightly reduced but still observable even in the absence of cholesterol in the PM-liposomes (Fig. 3d-f). These results indicate that cholesterol is required for $\mathrm{Ca}^{2+}$-dependent fusion of native vesicles, i.e., LDCV and SV, but not for liposome-liposome fusion.

\section{Membrane binding of synaptotagmin-1 independently of cholesterol.}

Cholesterol in the PM-liposomes is indispensable for $\mathrm{Ca}^{2+}$-dependent vesicle fusion, so we investigated whether the binding of synaptotagmin-1 to the $\mathrm{PIP}_{2}$-containing membrane is affected by cholesterol. The C2AB domain of synaptotagmin-1 ( Syt $\left._{97-421}\right)$ was labelled with Alexa Fluor 488 at S342C as a donor, and the PM-liposomes (Lip., protein-free) were labelled with Rhodamine (Rho)-PE as an acceptor (Online Methods). The $\mathrm{C} 2 \mathrm{AB}$ binding to liposomes was monitored by FRET between the C2AB domain (Alexa Fluor 488) and Rhodamine-labelled PM-liposomes (Fig. 4a). The C2AB domain of synaptotagmin-1 could bind to both cholesterol-containing and cholesterol-free liposomes in response to $\mathrm{Ca}^{2+}$, and the $\mathrm{Ca}^{2+}$ titration for $\mathrm{C} 2 \mathrm{AB}$ binding to the PM-liposomes $(0 \% \mathrm{Chol})$ was comparable to that of $25 \%$ Chol-containing PM-liposomes; this result demonstrates $\mathrm{Ca}^{2+}$-dependent $\mathrm{C} 2 \mathrm{AB}$ binding to anionic phospholipids regardless of cholesterol in liposomes (Fig. 4a-C). The PM-liposomes contain anionic phospholipids including $10 \% \mathrm{PS}, 4 \% \mathrm{PI}$, and $1 \% \mathrm{PIP}_{2}$, which provide complete coordination sites for the $\mathrm{Ca}^{2+}$-bound C2AB domain to interact with the membranes. (Fig. 4a-c).

To further assess membrane binding of the $C 2 A B$ domain, a fluorescence anisotropy measurement was conducted to monitor the rotational mobility of the C2AB domain, labelled with Alexa Fluor 488 at 342C; the PM-liposomes are label-free and protein-free (Fig. 4d). The C2AB binding to the PM-liposomes results in the increase of fluorescence anisotropy due to a reduction in the rotational mobility of the membranebound $\mathrm{C} 2 \mathrm{AB}$ domain (Fig. 4e). $\mathrm{Ca}^{2+}$ titration for membrane binding of the $\mathrm{C} 2 \mathrm{AB}$ domain showed no difference between the two sets of PM-liposomes (0\% vs $25 \%$ Chol) (Fig. $4 \mathrm{~d}-\mathrm{f})$. The increases of PIP 2 concentration in the PM-liposomes shifted $\mathrm{Ca}^{2+}$ titration curves to the left, which indicates an increase in the $\mathrm{Ca}^{2+}$-sensitivity of $\mathrm{C} 2 \mathrm{AB}$ membrane binding (Fig. $4 \mathrm{~g}$, Supplementary Fig. 3). High $\mathrm{PIP}_{2}$ concentration increases $\mathrm{Ca}^{2+}$ sensitivity for vesicle fusion ${ }^{31}$; this interaction implies that increase in the magnitude of the negative electrostatic potential in the plasma membranes increases its attraction of $\mathrm{Ca}^{2+}$-bound synaptotagmin-1. Even $5 \% \mathrm{PIP}_{2}$ in the PM-liposomes $\mathrm{Ca}^{2+}$ still failed to increase LDCV fusion without cholesterol (Fig. 4h). 
We have previously reported the membrane binding of the C2AB domain in a physiological ionic environment ${ }^{31}, 32$, showing that the $\mathrm{C} 2 \mathrm{AB}$ domain can only bind to the $\mathrm{PIP}_{2}$-containing plasma membrane, not to the SNARE complex. Then, we further validated if either the C2A or C2B domain still binds to vesicle membrane at physiological ionic strength. Indeed, the $\mathrm{C} 2 \mathrm{~A}$ and $\mathrm{C} 2 \mathrm{~B}$ domains bind to $\mathrm{V}$ liposomes ( $0 \% \mathrm{PIP}_{2}, 15 \% \mathrm{PS}$ included), but this interaction was completely disrupted at physiological ionic strength due to the charge-shielding effect of $\mathrm{Mg}^{2+}$ and ATP (Fig. 4i $)^{31,32}$. Both the C2A and C2B domains bind to the plasma membrane to drive vesicle fusion in a physiological ionic environment ${ }^{31,32}$, proposing that membrane binding of both the $\mathrm{C} 2 \mathrm{~A}$ and $\mathrm{C} 2 \mathrm{~B}$ domains might have cooperative and synergistic effect on membrane deformation. Taken together, $C 2 A B$ binding into the plasma membrane directly provides the driving force to lower the energy barrier for $\mathrm{Ca}^{2+}$-triggered vesicle fusion to occur. Cholesterol is only required for $\mathrm{Ca}^{2+}$-dependent vesicle fusion, but is not essential for $\mathrm{Ca}^{2+}$-dependent C2AB membrane binding.

\section{Membrane curvature is critical for $\mathrm{Ca}^{2+}$-dependent fusion.}

Despite the complete inhibition of $\mathrm{Ca}^{2+}$-dependent vesicle fusion (Figs. 2 and $\left.3 a, b\right)$, membrane binding of the $C 2 A B$ domain was still observable in the absence of cholesterol in liposomes (Fig. 4); this observation strongly suggests that the downstream of membrane binding of synaptotagmin- 1 is disrupted.

Hydrophobic residues in the $\mathrm{Ca}^{2+}$-binding loops of synaptotagmin-1 penetrate the inner leaflet of the plasma membrane $\mathrm{e}^{35}$, and probably lead to local membrane bending and deformation ${ }^{36,37}$. This local membrane deformation might accelerate vesicle fusion by lowering the energy barrier ${ }^{38}$. Cholesterol also regulates local membrane bending and deformation ${ }^{39}$, so in the next experiments we examined whether cholesterol has a critical function in membrane bending to trigger $\mathrm{Ca}^{2+}$-dependent vesicle fusion.

To this end we used different PM-liposomes of different sizes: large (LUV) with 110-nm diameter and small unilamellar vesicles (SUV) with 60-nm diameter (Online Methods, Supplementary Fig. 5). The average diameter of LDCVs is $150 \mathrm{~nm}$, ranging from $100 \mathrm{~nm}$ to $300 \mathrm{~nm}^{33}$. We expected that the synaptotagmin-1-induced changes in local membrane curvature and tension would be minimized in small liposomes (Fig. 5a,b), because they are already highly curved ${ }^{37}$. Indeed, $\mathrm{Ca}^{2+}$-dependent LDCV fusion was dramatically lower when SUVs were used compared to LUVs (Fig. 5c-e). The curvature effect on vesicle fusion was reproduced when the concentration of liposomes and the ratio of vesicle to liposome were changed (Supplementary Fig. 4a-d).

To further assess the effect of membrane curvature on vesicle fusion, we used SVs that have an average diameter of $45 \mathrm{~nm}^{40}$. As expected, $\mathrm{Ca}^{2+}$-dependent SV fusion with SUVs was completely impaired (Fig. 5f,g), whereas basal SV fusion was already saturated and augmented (Fig. $5 \mathrm{~g}$ ). Next, we replaced native SVs with small liposomes to confirm the curvature effect on vesicle fusion; small V-liposomes (SUV) that incorporate full length VAMP-2 and synaptotagmin-1 fuse with small PM-liposomes (SUV) either in the presence or absence of cholesterol (Supplementary Fig. 4e,f). Indeed, no $\mathrm{Ca}^{2+}$-dependent fusion was observed in case of small V-liposomes and small PM-liposomes, whereas LUVs show $\mathrm{Ca}^{2+}$ 
dependent liposome fusion (Fig. 3d-f). Altogether, high membrane tension and curvature elevate basal fusion, but decrease $\mathrm{Ca}^{2+}$-dependent vesicle fusion, because highly curved-membranes are likely to fuse without $\mathrm{Ca}^{2+}$ and show less change in membrane tension by membrane insertion of synaptotagmin-1.

\section{Cholesterol stabilizes membrane bending and deformation.}

The C2AB domain of synaptotagmin-1 has tubulation activity by membranes deformation and curvature generation ${ }^{36,37}$. Next, we performed a tubulation assay using negative stain TEM to test that cholesterol regulates local membrane bending and deformation induced by synaptotagmin-1 (Fig. 6). As expected, $\mathrm{Ca}^{2+} / \mathrm{C} 2 \mathrm{AB}$ domain induced tubulation by deforming membrane in the presence of cholesterol, whereas either $\mathrm{Ca}^{2+}$ or the $\mathrm{C} 2 \mathrm{AB}$ domain alone had no effect (Fig. 6a-d), as correlating with the previous reports ${ }^{36}$, 37. Intriguingly, we observed no tubulation activity of the $\mathrm{Ca}^{2+} / \mathrm{C} 2 \mathrm{AB}$ domain in the absence of cholesterol in the PM-liposomes (Fig. 6c,d). These TEM data provide evidence that cholesterol is crucial for stabilizing and strengthening membrane bending and deformation caused by synaptotagmin- 1 .

\section{Discussion}

Cholesterol regulates vesicle fusion as follows. First, it causes clustering of SNARE proteins in the plasma membrane. This clustering could increase the efficiency of membrane fusion ${ }^{29}, 30$. Cholesterol in the PMliposomes slightly increased the formation of SNARE complexes, and thus increased LDCV fusion (Fig. 2a-d). Second, cholesterol regulates the physical structure, fluidity, tension, and thickness of lipid membranes ${ }^{25}$. Cholesterol causes negative membrane curvature that might facilitate and promote vesicle fusion $^{13,25,41,42}$. Cholesterol also contributes to vesicle fusion by stabilizing fusion pores ${ }^{25,39,43,44}$. The plasma membrane deformations occur prior to vesicle fusion, and pre-fusion membrane curvature changes can be observed ${ }^{45}$. The high membrane bending energy can be released for fusion pore formation ${ }^{46}$. For examples, smaller vesicles, e.g., SUVs (Supplementary Fig. 4e,f) and SVs (Fig. 5f,g), have greater bending energy per unit of surface area and accordingly show robust basal fusion efficiency compared to large vesicles. Here, our data provide a novel model in which cholesterol mediates $\mathrm{Ca}^{2+}$ dependent vesicle fusion by stabilizing local membrane bending caused by synaptotagmin-1 binding. The curved plasma membrane has high bending energy, which can be released to drive fusion with vesicle membranes ${ }^{36,37,46}$. Thus, high membrane-bending energy stabilized by cholesterol lowers the energy barrier for vesicle fusion, thereby triggering $\mathrm{Ca}^{2+}$-dependent vesicle fusion (Fig. 7a,b).

The $\mathrm{C} 2 \mathrm{AB}$ domain of synaptotagmin- 1 is inserted into the plasma membrane ${ }^{35}$ and the membranebinding energy of the $C 2 A B$ domain is $\sim 18 \mathrm{k}_{\mathrm{B}} \mathrm{T}^{;}$; the binding leads to local membrane bending and deformation ${ }^{36,37}$. Membrane bending by synaptotagmin-1 could accelerate fusion by reducing the energy barrier. However, our data indicate that membrane bending by synaptotagmin-1 seems not enough to overcome the threshold of the energy barrier for fusion, when cholesterol is absent in the plasma membrane (Fig. 2,3, and 6). Cholesterol stabilizes and strengthens the plasma membrane deformation 
and bending caused by synaptotagmin-1 (Fig. 6d). This significant membrane bending energy can drive the membrane fusion (Fig. 7a,b).

Recently, Zuber's team observed membrane bending upon calcium influx in synaptosomes ${ }^{48}$. Using timeresolved cryo-electron tomography within 7 to $35 \mathrm{~ms}$ after diffusion of $52 \mathrm{mM} \mathrm{KCl}$ in synaptosomes, Zuber's group reported that fusion initiation occurs by membrane curvature ('buckling') of synaptic vesicle and the plasma membrane, showing $\mathrm{Ca}^{2+}$-dependent membrane bending before full fusion ${ }^{48}$. This cryo-electron tomography correlate with and strongly support our model that the $\mathrm{Ca}^{2+}$-bound $\mathrm{C} 2 \mathrm{AB}$ domain of synaptotagmin-1 induce membrane bending and curvature for vesicle fusion.

Our in-vitro reconstitution of vesicle fusion has an advantage of using purified native vesicles, i.e., LDCVs and SVs, and reproduces the physiological cholesterol effect on vesicle fusion, whereas V-liposomes fail (Fig. 3d-f). V-liposomes are independent of cholesterol for $\mathrm{Ca}^{2+}$-dependent fusion, in contrast to native vesicles (Fig. 2,3). V-liposomes might have some limitations to fully replace native vesicles for a fusion assay. Native vesicles differ from V-liposomes in lipid composition, protein density, contents diversity, and physical property; therefore the structural integrity of native vesicles allows them to mimic endogenous vesicle fusion in the in-vitro reconstitution. For example, we observed that native vesicles, LDCVs and SVs, remain stable and functionally active even after $3 \sim 5$ times freeze-thaw cycles ${ }^{31}, 33$. Despite $3 \sim 5$ times snap freeze-thaw cycles, biophysical and biochemical properties of purified LDCVs are preserved; i) the structure and size distribution of LDCVs are normal, ii) complete SNARE-dependent fusion of LDCVs using a lipid-mixing assay, and iii) acidification of LDCVs is fully functional ${ }^{33}$, suggesting that the membrane stability and rigidity of native vesicles after freeze-thaw cycles remain stable and functional. In contrast, proteoliposomes become immediately disrupted and destructed by freezing regardless of cholesterol in liposomes, thus these liposomes fail to undergo fusion (data not shown). Liposomes should not be frozen, because the freezing process fractures or ruptures liposomes, leading to destruction of liposome membranes; i.e., ice crystals generated by the freeze-thaw process cause liposome membranes to rupture. Ice crystal formation in the outer parts of liposomes can cause the interior of liposomes to expand until liposome membrane bursts.

However, vesicular proteins such as scaffold proteins and granins could stabilize vesicle structure and regulate membrane rigidity ${ }^{49}$. Native vesicles have high membrane rigidity compared to V-liposomes and native vesicles seem to require higher membrane bending energy than V-liposomes do, in order to overcome the barrier for fusion due to membrane fluidity and rigidity. The structural integrity of native vesicles can be advantages for the in-vitro reconstitution of vesicle fusion to reproduce physiological exocytosis, but the cause of the difference between native vesicles and liposomes for fusion efficiency, and the reproduction of physiological vesicle fusion remain topics for further study.

The molecular mechanisms of synaptotagmin-1 to trigger $\mathrm{Ca}^{2+}$-dependent fusion remain controversial; at least six different competing models have been proposed ${ }^{21}$. Synaptotagmin-1 mediates $\mathrm{Ca}^{2+}{ }^{2}$ dependent fusion by the electrostatic interaction, so several different synaptotagmin-1 models have been proposed, 
depending on the ionic environment ${ }^{32}$. Here we confirmed the cholesterol effect on vesicle fusion in a physiological ionic environment, i.e., normal ionic strength with $\mathrm{Mg}^{2+} / \mathrm{ATP}$. Both the $\mathrm{C} 2 \mathrm{~A}$ and $\mathrm{C} 2 \mathrm{~B}$ domains of synaptotagmin-1 are inserted into the plasma membrane (Fig. 4i) without interacting with SNARE proteins ${ }^{32}$, and therefore lead to membrane bending and deformation (Fig. 6). $\mathrm{Ca}^{2+}$ fails to trigger fusion in the absence of cholesterol despite the proteins, both SNARE assembly and membrane binding of synaptotagmin-1, being fully active and functional (Fig. 2,4$)$. Cholesterol has an critical function in $\mathrm{Ca}^{2+}$-dependent fusion, as an important lipid regulator by stabilizing membrane deformation and curvature caused by synaptotagmin-1.

Age-related cholesterol reduction is linked to reduced synaptic activity, and defects in synaptic transmission by cholesterol deficiency could result in neurodegeneration ${ }^{4}$. Our data explains the molecular mechanisms how cholesterol contributes to synaptic transmission and neuronal function and may pave the way for development of studies to explore to treatment of neurodegenerative and neurodevelopmental disorders by optimizing cholesterol levels in the plasma membrane.

\section{Declarations}

\section{Author Contributions}

Y.P., H.Y.A.M., and K.C.S purified vesicles and performed experiments. J.P. and S.M. conducted and analyzed TEM for liposomes and S.J.K. carried out TEM for chromaffin cells. J.K.R. did modelling. Y.P. collected and analyzed data. Y.P. wrote the manuscript and all authors read and provided their comments.

\section{Acknowledgements}

We thank Dr. Reinhard Jahn for constructs and samples. We are deeply indebted to Dr. Kyong-Tai Kim for technical assistanace. We thank Drs. Yongfeng Tong and Akshath Raghu Shetty from the Core Labs of the Qatar Environment and Energy Research Institute (QEERI) for technical support. Thanks to Dr. Ahmed Elalawy and Sarra Karrar from Widam Food Company for the arrangement of adrenal glands. This work was supported by the grant from Qatar Biomedical Research Institute (Project Number SF 2019004 to Y.P.).

\section{References}

1. Dietschy JM, Turley SD. Cholesterol metabolism in the brain. Curr Opin Lipido/ 12, 105-112 (2001).

2. Bjorkhem I, Meaney S. Brain cholesterol: long secret life behind a barrier. Arterioscler Thromb Vasc Bio/ 24, 806-815 (2004). 
3. Svennerholm L, Bostrom K, Jungbjer B, Olsson L. Membrane lipids of adult human brain: lipid composition of frontal and temporal lobe in subjects of age 20 to 100 years. Journal of neurochemistry 63, 1802-1811 (1994).

4. Martin M, Dotti CG, Ledesma MD. Brain cholesterol in normal and pathological aging. Biochim Biophys Acta 1801, 934-944 (2010).

5. Pfrieger FW. Cholesterol homeostasis and function in neurons of the central nervous system. Cell Mol Life Sci 60, 1158-1171 (2003).

6. Cartocci V, Servadio M, Trezza V, Pallottini V. Can Cholesterol Metabolism Modulation Affect Brain Function and Behavior? J Cell Physio/ 232, 281-286 (2017).

7. Martin MG, et al. Constitutive hippocampal cholesterol loss underlies poor cognition in old rodents. EMBO Mol Med 6, 902-917 (2014).

8. Linetti A, et al. Cholesterol reduction impairs exocytosis of synaptic vesicles. J Cell Sci 123, 595605 (2010).

9. Liu Q, et al. Neuronal LRP1 knockout in adult mice leads to impaired brain lipid metabolism and progressive, age-dependent synapse loss and neurodegeneration. The Journal of neuroscience : the official journal of the Society for Neuroscience 30, 17068-17078 (2010).

10. Lange Y, Steck TL. Active membrane cholesterol as a physiological effector. Chem Phys Lipids 199, 74-93 (2016).

11. Murray DH, Tamm LK. Clustering of syntaxin-1A in model membranes is modulated by phosphatidylinositol 4,5-bisphosphate and cholesterol. Biochemistry 48, 4617-4625 (2009). 
12. Sieber JJ, et al. Anatomy and dynamics of a supramolecular membrane protein cluster. Science 317, 1072-1076 (2007).

13. Zhang J, Xue R, Ong WY, Chen P. Roles of cholesterol in vesicle fusion and motion. Biophys J 97, 1371-1380 (2009).

14. Churchward MA, Rogasevskaia T, Hofgen J, Bau J, Coorssen JR. Cholesterol facilitates the native mechanism of Ca2+-triggered membrane fusion. J Cell Sci 118, 4833-4848 (2005).

15. Mailman T, Hariharan M, Karten B. Inhibition of neuronal cholesterol biosynthesis with lovastatin leads to impaired synaptic vesicle release even in the presence of lipoproteins or geranylgeraniol. Journal of neurochemistry 119, 1002-1015 (2011).

16. Teixeira G, Vieira LB, Gomez MV, Guatimosim C. Cholesterol as a key player in the balance of evoked and spontaneous glutamate release in rat brain cortical synaptosomes. Neurochemistry international 61, 1151-1159 (2012).

17. Mercer AJ, Szalewski RJ, Jackman SL, Van Hook MJ, Thoreson WB. Regulation of presynaptic strength by controlling $\mathrm{Ca} 2+$ channel mobility: effects of cholesterol depletion on release at the cone ribbon synapse. Journal of neurophysiology 107, 3468-3478 (2012).

18. Tarakanova OI, Petrov AM, Zefirov AL. The role of membrane cholesterol in neurotransmitter release from motor nerve terminals. Doklady biological sciences : proceedings of the Academy of Sciences of the USSR, Biological sciences sections / translated from Russian 438, 138-140 (2011).

19. Jahn R, Scheller RH. SNAREs-engines for membrane fusion. Nat Rev Mol Cell Biol 7, 631-643 (2006). 
20. Brunger AT, Choi UB, Lai Y, Leitz J, Zhou Q. Molecular Mechanisms of Fast Neurotransmitter Release. Annu Rev Biophys 47, 469-497 (2018).

21. Park Y, Ryu JK. Models of synaptotagmin-1 to trigger $\mathrm{Ca}(2+)$-dependent vesicle fusion. FEBS Lett 592, 3480-3492 (2018).

22. Anderson RG. The caveolae membrane system. Annual review of biochemistry $67,199-225$ (1998).

23. Allender DW, Sodt AJ, Schick M. Cholesterol-Dependent Bending Energy Is Important in Cholesterol Distribution of the Plasma Membrane. Biophys J 116, 2356-2366 (2019).

24. Wang W, Yang L, Huang HW. Evidence of cholesterol accumulated in high curvature regions: implication to the curvature elastic energy for lipid mixtures. Biophys J 92, 2819-2830 (2007).

25. Yang ST, Kreutzberger AJB, Lee J, Kiessling V, Tamm LK. The role of cholesterol in membrane fusion. Chem Phys Lipids 199, 136-143 (2016).

26. Hubert M, Larsson E, Lundmark R. Keeping in touch with the membrane; protein- and lipidmediated confinement of caveolae to the cell surface. Biochem Soc Trans 48, 155-163 (2020).

27. Moren B, et al. EHD2 regulates caveolar dynamics via ATP-driven targeting and oligomerization. Mol Biol Cell 23, 1316-1329 (2012).

28. Rothberg KG, Heuser JE, Donzell WC, Ying YS, Glenney JR, Anderson RG. Caveolin, a protein component of caveolae membrane coats. Cell 68, 673-682 (1992).

29. Chamberlain LH, Burgoyne RD, Gould GW. SNARE proteins are highly enriched in lipid rafts in PC12 cells: implications for the spatial control of exocytosis. Proceedings of the National Academy of Sciences 
of the United States of America 98, 5619-5624 (2001).

30. Lang $T$, et al. SNAREs are concentrated in cholesterol-dependent clusters that define docking and fusion sites for exocytosis. EMBO J 20, 2202-2213 (2001).

31. Park Y, et al. Controlling synaptotagmin activity by electrostatic screening. Nature structural \& molecular biology 19, 991-997 (2012).

32. Park Y, et al. Synaptotagmin-1 binds to PIP(2)-containing membrane but not to SNAREs at physiological ionic strength. Nature structural \& molecular biology 22, 815-823 (2015).

33. Birinci Y, Preobraschenski J, Ganzella M, Jahn R, Park Y. Isolation of large dense-core vesicles from bovine adrenal medulla for functional studies. Sci Rep 10, 7540 (2020).

34. Pobbati AV, Stein A, Fasshauer D. N- to C-terminal SNARE complex assembly promotes rapid membrane fusion. Science 313, 673-676 (2006).

35. Herrick DZ, Sterbling S, Rasch KA, Hinderliter A, Cafiso DS. Position of synaptotagmin I at the membrane interface: cooperative interactions of tandem C2 domains. Biochemistry 45, 9668-9674 (2006).

36. Martens S, Kozlov MM, McMahon HT. How synaptotagmin promotes membrane fusion. Science 316, 1205-1208 (2007).

37. Hui E, Johnson CP, Yao J, Dunning FM, Chapman ER. Synaptotagmin-mediated bending of the target membrane is a critical step in $\mathrm{Ca}(2+)$-regulated fusion. Cell 138, 709-721 (2009). 
38. Kozlov MM, Chernomordik LV. The protein coat in membrane fusion: lessons from fission. Traffic 3, 256-267 (2002).

39. Ivankin A, Kuzmenko I, Gidalevitz D. Cholesterol mediates membrane curvature during fusion events. Phys Rev Lett 108, 238103 (2012).

40. Takamori S, et al. Molecular anatomy of a trafficking organelle. Cell 127, 831-846 (2006).

41. Chernomordik L, Kozlov MM, Zimmerberg J. Lipids in biological membrane fusion. J Membr Biol 146, 1-14 (1995).

42. Chen Z, Rand RP. The influence of cholesterol on phospholipid membrane curvature and bending elasticity. Biophys J 73, 267-276 (1997).

43. Kreutzberger AJ, Kiessling V, Tamm LK. High cholesterol obviates a prolonged hemifusion intermediate in fast SNARE-mediated membrane fusion. Biophys J 109, 319-329 (2015).

44. Wu L, Courtney KC, Chapman ER. Cholesterol stabilizes recombinant exocytic fusion pores by altering membrane bending rigidity. Biophys J 120, 1367-1377 (2021).

45. Anantharam A, Axelrod D, Holz RW. Real-time imaging of plasma membrane deformations reveals pre-fusion membrane curvature changes and a role for dynamin in the regulation of fusion pore expansion. Journal of neurochemistry 122, 661-671 (2012).

46. Zhang Z, Jackson MB. Membrane bending energy and fusion pore kinetics in $\mathrm{Ca}(2+)$-triggered exocytosis. Biophys J 98, 2524-2534 (2010). 
47. Gruget $\mathrm{C}$, et al. Synaptotagmin-1 membrane binding is driven by the C2B domain and assisted cooperatively by the C2A domain. Sci Rep 10, 18011 (2020).

48. Radecke J, et al. Morphofunctional changes at the active zone during synaptic vesicle exocytosis. bioRxiv, 2022.2003.2007.483217 (2022).

49. Prinz WA, Hinshaw JE. Membrane-bending proteins. Crit Rev Biochem Mol Bio/ 44, 278-291 (2009).

50. Ahmed S, Holt M, Riedel D, Jahn R. Small-scale isolation of synaptic vesicles from mammalian brain. Nature protocols 8, 998-1009 (2013).

51. Radhakrishnan A, Stein A, Jahn R, Fasshauer D. The Ca2+ affinity of synaptotagmin 1 is markedly increased by a specific interaction of its C2B domain with phosphatidylinositol 4,5-bisphosphate. $J$ Biol Chem 284, 25749-25760 (2009).

52. Nalefski EA, Falke JJ. Use of fluorescence resonance energy transfer to monitor $\mathrm{Ca}(2+)$-triggered membrane docking of C2 domains. Methods in molecular biology 172, 295-303 (2002).

53. Park YS, et al. Involvement of protein kinase C-epsilon in activity-dependent potentiation of large dense-core vesicle exocytosis in chromaffin cells. The Journal of neuroscience : the official journal of the Society for Neuroscience 26, 8999-9005 (2006).

54. Park Y, et al. alpha-SNAP interferes with the zippering of the SNARE protein membrane fusion machinery. J Biol Chem 289, 16326-16335 (2014).

\section{Online Methods}

Purification of large dense-core vesicles (LDCVs) and synaptic vesicles (SVs). LDCVs, also known as chromaffin granules, were purified from bovine adrenal medullae using continuous sucrose gradient and resuspended with fusion buffer containing 120 mM K-glutamate, 20 mM K-acetate, and 20 mM 
HEPES.KOH, pH 7.4, as elsewhere ${ }^{33}$. SV from mouse brains were purified as described elsewhere ${ }^{50}$. Briefly, mice brains were homogenized in homogenization buffer supplemented with protease inhibitors, using a glass-Teflon homogenizer. The homogenate was centrifuged for $10 \mathrm{~min}$ at $1,000 \mathrm{~g}$ and the resulting supernatant was further centrifuged for $15 \mathrm{~min}$ at $15,000 \mathrm{~g}$. The synaptosome pellet was lysed by adding ice-cold water, followed by centrifugation for $25 \mathrm{~min}$ at $48,000 \mathrm{~g}$. The resulting supernatant was overlaid onto a $0.7 \mathrm{M}$ sucrose cushion and centrifuged for $1 \mathrm{~h}$ at $133,000 \mathrm{~g}$. The pellet was resuspended in fusion buffer (120 mM K-glutamate, 20 mM K-acetate, 20 mM HEPES.KOH, pH 7.4).

Protein purification. All SNARE and the C2AB domain of synaptotagmin-1 constructs based on rat sequences were expressed in E. coli strain BL21 (DE3) and purified by $\mathrm{Ni}^{2+}-\mathrm{NTA}$ affinity chromatography followed by ion-exchange chromatography as described elsewhere ${ }^{31,32}$. The stabilized Q-SNARE complex consisting syntaxin-1A (aa 183-288), SNAP-25A (no cysteine, cysteines replaced by alanines) in a 1:1 ratio by the C-terminal VAMP-2 fragment (aa 49-96) was purified as described earlier ${ }^{34}$. The binary QSNARE complex containing the full-length syntaxin-1A (1-288) and SNAP-25A (no cysteine, cysteines replaced by alanines) was expressed using co-transformation ${ }^{31}$. The full-length VAMP-2, soluble cytoplasmic region of VAMP-2 (VAMP-2 ${ }_{1-96}$ ), full-length synaptotagmin-1, C2AB domain of synaptotagmin-1 (aa 97-421), C2A domain (aa 96-262), C2B domain (aa 248-421), and C2ab mutant (D178A, D230A, D232A, D309A, D363A, D365A) were purified by Mono S column (GE Healthcare, Piscataway, NJ) as described previously ${ }^{51}$. The stabilized Q-SNARE complex and the syntaxin-1A/SNAP25A binary SNARE complex were purified by $\mathrm{Ni}^{2+}{ }^{2}$ NTA affinity chromatography followed by ion-exchange chromatography on a Mono Q column (GE Healthcare, Piscataway, NJ) in the presence of $50 \mathrm{mM}$ n-octyl$\beta$-D-glucoside $(O G)^{31}$. The point mutated C2AB domain (S342C) was labelled with Alexa Fluor 488 C5 maleimide $\left(\mathrm{C} 2 \mathrm{AB}{ }^{\mathrm{A} 488}\right)^{51}$.

Lipid composition of liposomes. Lipid composition (molar percentages) of the PM-liposomes that contain the Q-SNARE complex consists of 45\% PC (L-a-phosphatidylcholine, Cat. 840055), 15\% PE (L-aphosphatidylethanolamine, Cat. 840026), 10\% PS (L-a-phosphatidylserine, Cat. 840032), 25\% Chol (cholesterol, Cat. 700000), 4\% PI (L-a-phosphatidylinositol, Cat. 840042), and 1\% PI(4,5) $\mathrm{P}_{2}$ (Cat. 840046). When cholesterol was excluded ( $0 \% \mathrm{Chol}), \mathrm{PC}$ contents were accordingly adjusted. In case of changing $\mathrm{PI}(4,5) \mathrm{P}_{2}$ concentration, $\mathrm{PI}$ contents were accordingly adjusted. VAMP-2/synaptotagmin-1containing V-liposomes are composed of $55 \%$ PC, $20 \%$ PE, $15 \%$ PS, and $10 \%$ Chol. For FRET-based lipidmixing assays, 1.5\% 1,2-dioleoyl-sn-glycero-3-phosphoethanolamine-N-(7-nitrobenz-2-oxa-1,3-diazol-4-yl (NBD-DOPE) and 1.5\% 1,2-dioleoyl-sn-glycero-3-phosphoethanolamine-N-lissamine rhodamine B sulfonyl ammonium salt (Rhodamine-DOPE) were incorporated in the PM-liposomes (accordingly $12 \%$ unlabeled $\mathrm{PE})$ as a donor and an acceptor dye, respectively. For FRET measurement using $C 2 \mathrm{AB}^{\mathrm{A} 488}$, $1.5 \%$ Rhodamine-DOPE was included in the PM-liposomes (protein-free). In the case of FRET for 
tryptophan, 5\% N-(5-dimethylaminonaphthalene-1-dulfonyl)-1,2-dihexadecanoyl-sn-glycero-3phosphoethanolamine, triethylammonium salt (dansyl-DHPE) was incorporated in the PM-liposomes (protein-free). All lipids were from Avanti Polar lipids except dansyl-DHPE (Invitrogen).

Preparation of proteoliposomes. Incorporation of the stabilized and binary Q-SNARE complex into large unilamellar vesicles (LUVs, $110 \mathrm{~nm}$ in diameter) was achieved by OG-mediated reconstitution, called the direct method, i.e. incorporation of proteins into preformed liposomes ${ }^{31,32}$. Briefly, lipids dissolved in a 2:1 chloroform-methanol solvent were mixed according to lipid composition. The solvent was removed using a rotary evaporator (which generated lipid film on a glass flask), then lipids were resuspended in 1.5 $\mathrm{mL}$ diethyl ether and $0.5 \mathrm{~mL}$ buffer containing $150 \mathrm{mM} \mathrm{KCl}$ and $20 \mathrm{mM} \mathrm{HEPES} / \mathrm{KOH} \mathrm{pH} \mathrm{7.4.} \mathrm{After}$ sonication on ice ( $3 \times 45 \mathrm{~s}$ ), multilamellar vesicles were prepared by reverse-phase evaporation using a rotary evaporator as diethyl ether was removed. Multilamellar vesicles $(0.5 \mathrm{~mL})$ were then extruded using polycarbonate membranes of pore size $100 \mathrm{~nm}$ (Avanti Polar lipids) to give uniformly-distributed LUVs with the average diameter of $110 \mathrm{~nm}$ (Supplementary Fig. 5). After the preformed LUVs had been prepared, SNARE proteins or the full-length VAMP-2/synaptotagmin-1 were incorporated into them using $O G$, a mild non-ionic detergent, then the $O G$ was removed by dialysis overnight in $1 \mathrm{~L}$ buffer containing $150 \mathrm{mM} \mathrm{KCl}$ and $20 \mathrm{mM} \mathrm{HEPES} / \mathrm{KOH} \mathrm{pH} 7.4$ together with $2 \mathrm{~g} \mathrm{SM}-2$ adsorbent beads.

To make small unilamellar vesicles (SUVs) using the direct method, 110-nm LUVs produced as described above extruded through polycarbonate membranes with 50-nm pore size (yielding SUV that had average diameter of $60 \mathrm{~nm}$, Supplementary Fig. 5). After preparing preformed SUVs, protein incorporation was completed by OG as described for LUV. The size distribution of proteoliposomes was determined using dynamic light scattering (DLS) (Supplementary Fig. 5). Proteoliposomes have protein-to-lipid molar ratio of $1: 500(n / n)$.

Vesicle fusion assay. A FRET-based lipid-mixing assay was applied to monitor vesicle fusion in vitro 31,32 . LDCV or SV fusion reactions were performed at $37^{\circ} \mathrm{C}$ in $1 \mathrm{~mL}$ fusion buffer containing $120 \mathrm{mM} \mathrm{K}$ glutamate, $20 \mathrm{mM} \mathrm{K}$-acetate, $20 \mathrm{mM} \mathrm{HEPES-KOH} \mathrm{(pH} \mathrm{7.4),} 1 \mathrm{mM} \mathrm{MgCl}_{2}$, and $3 \mathrm{mM}$ ATP. ATP should be made freshly before experiments, because ATP is easily destroyed by freezing and thawing. Free $\mathrm{Ca}^{2+}$ concentration in the presence of ATP and $\mathrm{Mg}^{2+}$ was calibrated using Maxchelator simulation program. The fluorescence dequenching signal was measured using Fluoromax (Horiba Jobin Yvon) with wavelengths of $460 \mathrm{~nm}$ for excitation and $538 \mathrm{~nm}$ for emission. Fluorescence values were normalized as a percentage of maximum donor fluorescence (total fluorescence) after addition of $0.1 \%$ Triton X-100 at the end of experiments. 
Fluorescence resonance energy transfer (FRET). The C2AB domain of synaptotagmin-1 (30 nM, S342C) was labeled with Alexa Fluor 488, a donor dye. C2AB fragment was engineered to contain a single Cys residue (S342C) and labelled with Alexa Fluor 488. 1.5\% Rhodamine-DOPE (Rho-PE), incorporated in the PM-liposomes (protein-free), was used as an acceptor. Unless otherwise stated, liposomes were LUVs prepared by the direct method. Donor fluorescence signal was measured at $37^{\circ} \mathrm{C}$ using Fluoromax (Horiba Jobin Yvon) with wavelengths of $488 \mathrm{~nm}$ for excitation and $516 \mathrm{~nm}$ for emission in $1 \mathrm{~mL}$ fusion buffer containing $120 \mathrm{mM}$ K-glutamate, $20 \mathrm{mM}$ K-acetate, $20 \mathrm{mM} \mathrm{HEPES}-\mathrm{KOH}$ (pH 7.4), $1 \mathrm{mM} \mathrm{MgCl}_{2}$, and $3 \mathrm{mM}$ ATP. FRET was normalized as net changes of donor fluorescence intensity and C2AB binding was presented as percentage of maximum $\mathrm{C} 2 \mathrm{AB}$ binding induced by $1 \mathrm{mM} \mathrm{Ca}^{2+}$. In Supplementary Figure 3, FRET was normalized as $F / F_{0}$, where $F_{0}$ represents the initial value of the donor fluorescence intensity.

Liposome binding of the C2AB domain was also monitored using the tryptophan-dansyl FRET pair as a donor-acceptor dye in which dansyl-DHPE incorporated in liposomes leads to quenching of fluorescence emitted from tryptophan of the C2AB domain ${ }^{52}$. Then $1 \mathrm{mM} C 2 \mathrm{AB}, 3 \mathrm{mM}$ C2A, or $3 \mathrm{mM} C 2 \mathrm{~B}$ was incubated with V-liposomes (protein-free). Donor fluorescence signal was measured at $37^{\circ} \mathrm{C}$ using Fluoromax (Horiba Jobin Yvon) with wavelengths of $295 \mathrm{~nm}$ for excitation and $350 \mathrm{~nm}$ for emission in 1 $\mathrm{mL}$ fusion buffer containing $120 \mathrm{mM} \mathrm{K}$-glutamate, $20 \mathrm{mM} \mathrm{K}$-acetate, $20 \mathrm{mM} \mathrm{HEPES}-\mathrm{KOH}$ (pH 7.4), $1 \mathrm{mM}$ $\mathrm{MgCl}_{2}$, and $3 \mathrm{mM}$ ATP. FRET monitoring $\mathrm{C} 2 \mathrm{AB}$ binding was normalized as a percentage of $\left(\mathrm{F}_{0}-\mathrm{F}\right) / \mathrm{F}_{0}$.

Fluorescence anisotropy measurements. The C2AB fragment (20 nM, S342C) were labelled with Alexa Fluor $488^{51}$. Anisotropy was measured in a Fluorolog (Horiba Jobin Yvon) at $37^{\circ} \mathrm{C}$ in $1 \mathrm{ml}$ of buffer containing $120 \mathrm{mM} \mathrm{K}$-glutamate, $20 \mathrm{mM} \mathrm{K}$-acetate, and $20 \mathrm{mM} \mathrm{HEPES}-\mathrm{KOH}$ (pH 7.4), $1 \mathrm{mM} \mathrm{MgCl}_{2}$, and 3 mM ATP. Excitation wavelength was $495 \mathrm{~nm}$, and emission was measured at $520 \mathrm{~nm}$. Lipid composition of the PM-liposomes (protein-free) was identical to those used in a fusion assay except labelled PE (45\% PC, $15 \%$ PE, $10 \%$ PS, $25 \%$ Chol, $4 \% \mathrm{PI}$, and $1 \%$ PIP2). In the case of $0 \%$ Chol, PC contents were adjusted accordingly $(70 \% \mathrm{PC})$.

Ternary SNARE complex formation assay. Tetanus neurotoxin (TeNT) degrades free VAMP-2 whereas VAMP-2, assembled in the ternary SNARE complex, is resistant to TeNT ${ }^{31}$. After incubation of LDCVs with the PM-liposomes ( $0 \%$ or $25 \% \mathrm{Chol}$ ) that contain the stabilized Q-SNARE complex for $20 \mathrm{~min}$ at $37^{\circ} \mathrm{C}$ without $\mathrm{Ca}^{2+}$, the sample was subjected to TeNT treatment $\left(200 \mathrm{nM}, 30 \mathrm{~min}, 37^{\circ} \mathrm{C}\right)$ then boiled for $5 \mathrm{~min}$ at $95^{\circ} \mathrm{C}$ and analysed by immunoblotting with antibody against VAMP-2 (clone number 69.1, Synaptic Systems (Göttingen, Germany)). 
Preparation of bovine chromaffin cells. Chromaffin cells were isolated from the bovine adrenal gland medulla by two-step collagenase digestion as previously described ${ }^{53}$. The cells were grown on poly- ${ }^{-}{ }^{-}$ lysine-coated glass coverslips in Dulbecco's modified Eagle medium/F-12 (Invitrogen, CA) containing 10\% fetal bovine serum (Hyclone Laboratories, UT) and 1\% antibiotics (Invitrogen, CA).

Amperometric measurement. Recordings of LDCV exocytosis from chromaffin cells were performed as described previously ${ }^{53}$. Carbon-fiber electrodes were fabricated from $8 \mu \mathrm{m}$ diameter carbon fibers and back-filled with $3 \mathrm{M} \mathrm{KCl}$. The amperometric current, generated by oxidation of catecholamine, was measured using an axopatch 200B amplifier (Axon Instruments Inc., CA), which was operated in voltageclamp mode at a holding potential of $+650 \mathrm{mV}$. Amperometric signals were low-pass filtered at $1 \mathrm{kHz}$ and sampled at $500 \mathrm{~Hz}$. For data acquisition and analysis, pCLAMP 11 software (Axon Instruments) was used. The area of amperometric current represents the total amount of released catecholamine. Amperometric current generated by repetitive stimulation was individually integrated. Relative exocytosis is presented as a percentage of the first DMPP-induced total catecholamine release.

Transmission electron microscopy (TEM). Chromaffin cells, grown on Vitrogen collagen matrix (Cohesion, Palo Alto, CA), were washed out with Locke's solution containing $157.4 \mathrm{mM} \mathrm{NaCl}, 5.6 \mathrm{mM} \mathrm{KCl}, 2.2 \mathrm{mM}$ $\mathrm{CaCl}_{2}, 1.2 \mathrm{mM} \mathrm{MgCl}_{2}, 5.6 \mathrm{mM}$ D-glucose, $5 \mathrm{mM} \mathrm{HEPES}$, and $3.6 \mathrm{mM} \mathrm{NaHCO}_{3}, \mathrm{pH} 7.4$ titrated by $\mathrm{NaOH}$. As described previously ${ }^{53}$, cells were fixed with $2 \%$ paraformaldehyde and $2 \%$ glutaraldehyde in $0.05 \mathrm{M}$ sodium cacodylate buffer at $\mathrm{pH} 7.4$ for $20 \mathrm{~min}$ at room temperature. Cells were post-fixed with $0.5 \%$ osmium tetroxide in $0.05 \mathrm{M}$ sodium cacodylate buffer at $\mathrm{pH} 7.4$ for $30 \mathrm{~min}$ at room temperature. Cells were further dehydrated in graded ethanol solutions and embedded in LR White resin (London Resin Co., Berkshire, UK). Silver-gold thin sections were stained with uranyl acetate and lead citrate. The thin sections were examined under JEOL 1200 EX2 transmission electron microscope at $80 \mathrm{kV}$.

For liposomes, $5 \mu \mathrm{L}$ of samples were deposited on carbon-coated 400-mesh copper grids (CF400-CU, Electron Microscopy Sciences). Grids were stained with uranyl acetate for negative staining and embedded in methylcellulose-uranyl acetate. Liposomes were visualized at $80 \mathrm{kV}$ in Talos F200C Transmission Electron Microscope (Thermo Fisher Scientific). The images were acquired using bottommounted CETA camera.

Liposome co-flotation assay. Liposomes float up through the gradient due to their buoyancy and free proteins remain in the bottom of the gradient, whereas proteins incorporated in liposomes co-float to the buoyant density of the liposomes ${ }^{54}$. First, $30 \mu \mathrm{L}$ of liposomes that incorporate the stabilized Q-SNARE complex were mixed with Nycodenz (Axis Shield, $80 \%, 30 \mu \mathrm{L}$ ) and a second Nycodenz layer $(30 \%, 40 \mu \mathrm{L}$ ) was gently applied followed by another layer of buffer $(40 \mu \mathrm{L})$. The density gradient was centrifuged 
using a Beckman TL-100 ultracentrifuge (TLS55 rotor, 100,000g, $4^{\circ} \mathrm{C}, 1 \mathrm{~h}$ ). The $20-\mu \mathrm{L}$ aliquots were carefully taken from the top of the gradient and analysed by coomassie blue staining.

Statistical analysis. The statistical difference between two groups was evaluated by Student's t-test using GraphPad Prism. Probabilities of $p<0.05$ were considered significant.

\section{Figures}

\section{Figure 1}
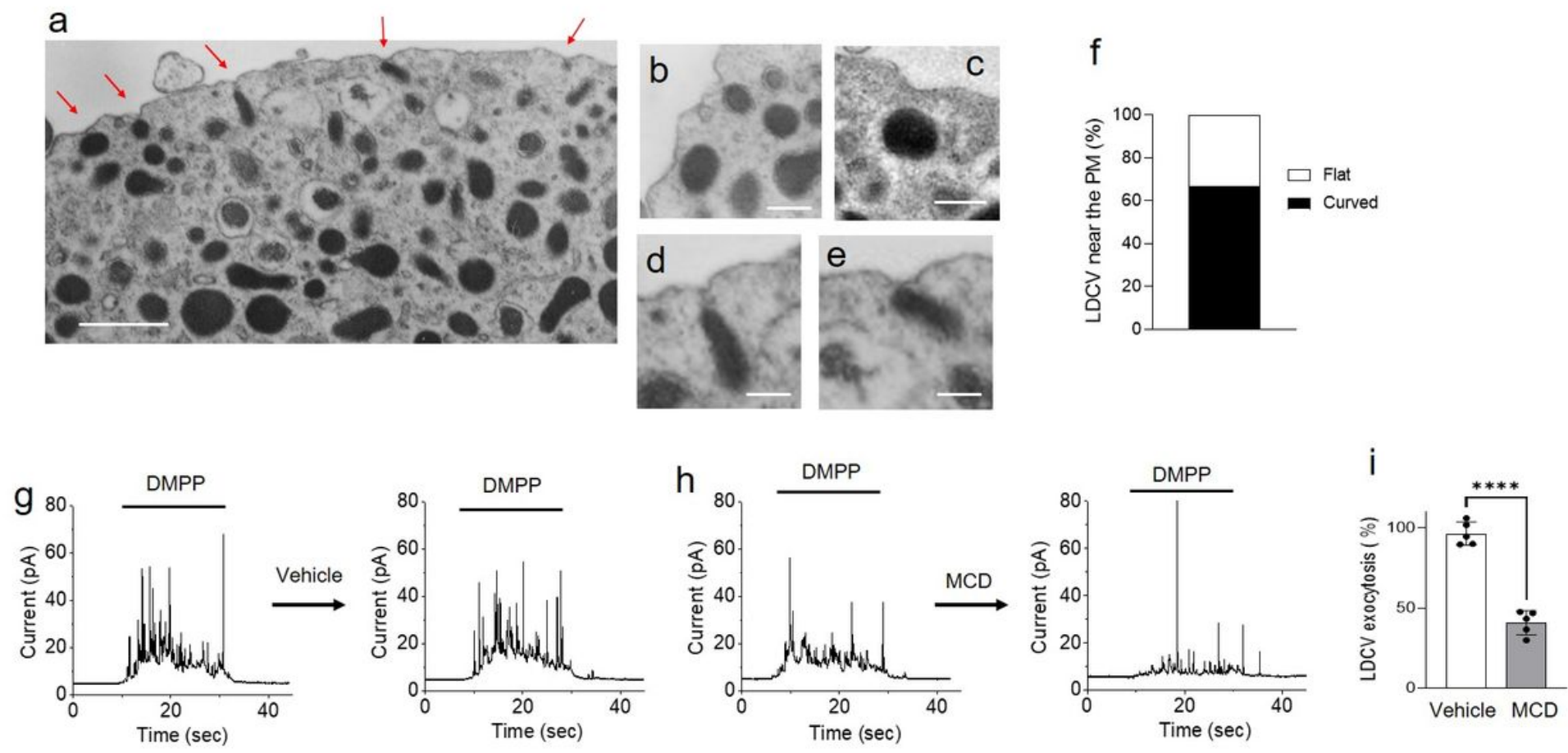

\section{Figure 1}

Cholesterol depletion inhibits LDCV exocytosis in chromaffin cells. (a) Transmission electron microscope (TEM) image of chromaffin cells showing invagination of the plasma membrane (PM) into LDCVs. Scale, $500 \mathrm{~nm}$. (b-e) Magnified TEM images of LDCVs. Scale, $200 \mathrm{~nm}$. (f) LDCVs close to the PM less than 100 $\mathrm{nm}$ distance between LDCV and PM were counted; LDCVs near either flat or curved PM is presented as a percentage (total $36 \mathrm{LDCV}$ s proximal to the PM from three independent experiments). (g-i) LDCV exocytosis in chromaffin cells measured by amperometry. $M C D\left(10 \mathrm{mM}, 2 \mathrm{~h}, 37^{\circ} \mathrm{C}\right)$ reduced LDCV exocytosis of chromaffin cells $(\mathbf{h})$. $(\mathbf{g}, \mathbf{h})$ Shown are typical amperometric traces upon DMPP stimulations finally activating voltage-gated calcium channel for $20 \mathrm{sec}$. Chromaffin cells were treated with either vehicle (g) or MCD (h) for $2 \mathrm{~h}$ after the first DMPP stimulation. (i) Amperometric current generated by repetitive stimulation was individually integrated. Relative LDCV exocytosis is presented as a percentage 
of the first DMPP-induced total catecholamine release. Data are means \pm SD from 5 independent experiments. ${ }^{\star \star * *}, p<0.0001$.

Figure 2
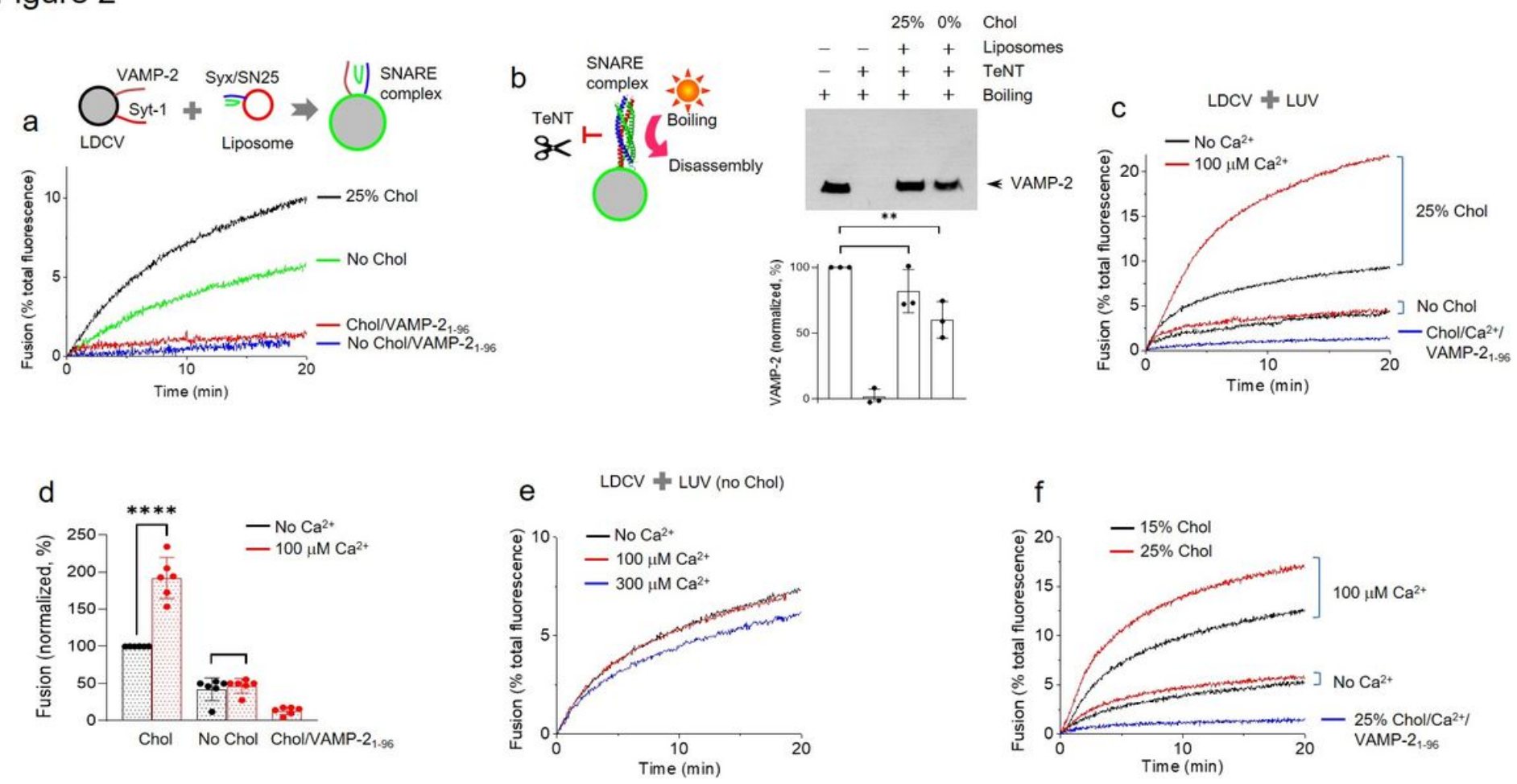

Figure 2

Cholesterol is required for $\mathrm{Ca}^{2+}$-triggered vesicle fusion. (a) In-vitro reconstitution of large dense-core vesicle (LDCV) fusion using a lipid-mixing assay. Purified LDCVs were incubated with PM-liposomes that incorporate the stabilized Q-SNARE complex of syntaxin-1A/SNAP-25A (Syx/SN25) in a 1:1 molar ratio (Online Methods). Cholesterol (Chol) was included either $25 \%$ or $0 \%$ in the PM-liposomes. For clarity, only endogenous VAMP-2 and synaptotagmin-1 of native LDCVs are shown and full fusion is presented. (b) Formation of the ternary SNARE complex after vesicle fusion in the presence or absence of cholesterol in the PM-liposomes. LDCVs were incubated with liposomes for 20 min without $\mathrm{Ca}^{2+}$, then treated with Tetanus neurotoxin (TeNT). TeNT-resistant VAMP-2 indicates the ternary SNARE complex formation in SDS-PAGE (Online Methods). Boiling at $95^{\circ} \mathrm{C}$ disrupts the ternary SNARE complex so that VAMP-2 migrates to its size. Data are mean \pm SD from 3 independent experiments $(\mathrm{n}=3)$. **, $p<0.01$. (c,d) Dependence on cholesterol for $\mathrm{Ca}^{2+}$-dependent vesicle fusion. Addition of $100 \mu \mathrm{M}$ free $\mathrm{Ca}^{2+}$ provoked fusion of LDCV with the PM-liposomes. Preincubation of the PM-liposomes with VAMP-2 ${ }_{1-96}$ caused competitive inhibition that blocked SNARE-mediated fusion. Fusion is presented as a percentage of basal fusion with Chol-containing PM-liposomes in the absence of $\mathrm{Ca}^{2+}$. Data are mean \pm SD $(n=6$ independent experiments). ${ }^{* * *}, p<0.0001$. (e) Free $\mathrm{Ca}^{2+}$ was increased to $300 \mu \mathrm{M}$, when LDCVs fused with the PM-liposomes ( $0 \%$ Chol). (f) Liposomes contained either $15 \%$ or $25 \%$ of Chol. Lipid composition of the PM-liposomess: $45 \%$ PC, $15 \%$ PE, $10 \%$ PS, $25 \%$ Chol, 4\% PI, and 1\% PI $(4,5) \mathrm{P}_{2}$. When Chol was 
reduced, PC contents were adjusted accordingly. Physiological ionic strength and $1 \mathrm{mM} \mathrm{MgCl} / 3 \mathrm{mM}$ ATP were included in all experiments.

\section{Figure 3}
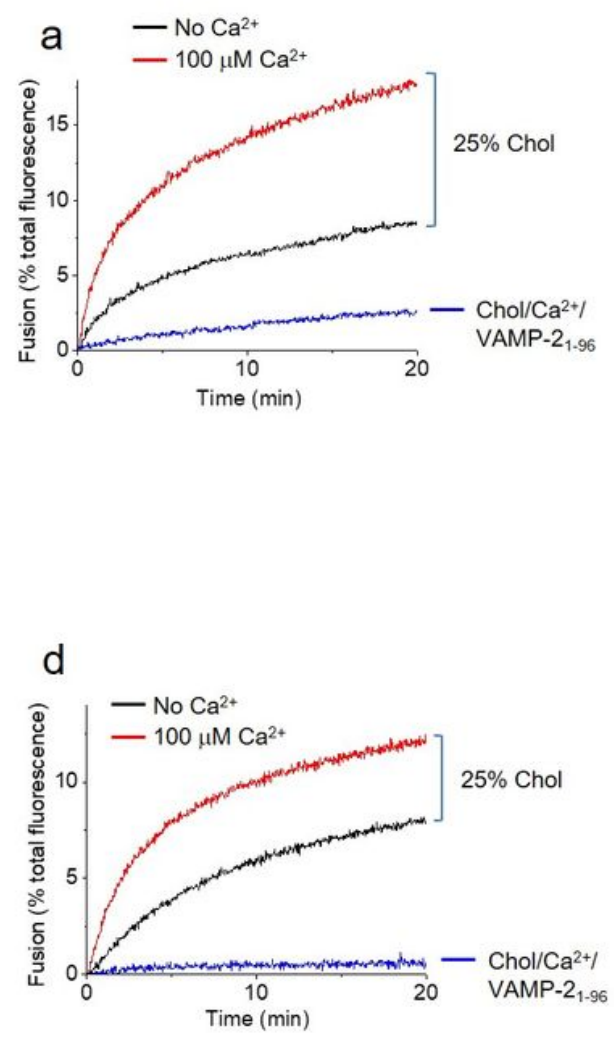
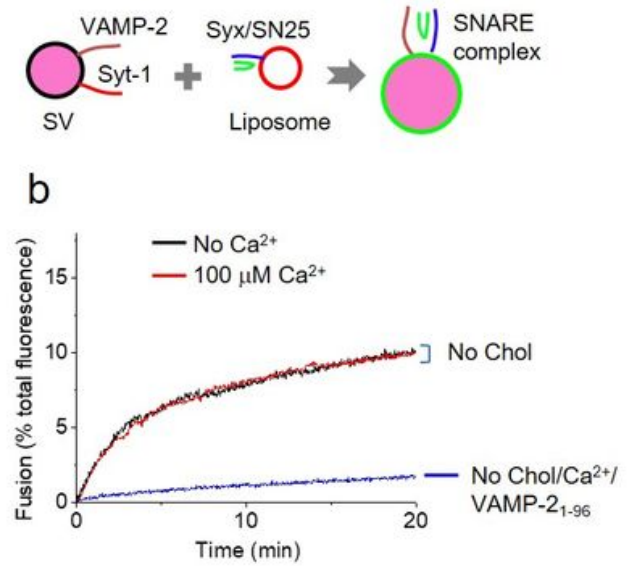
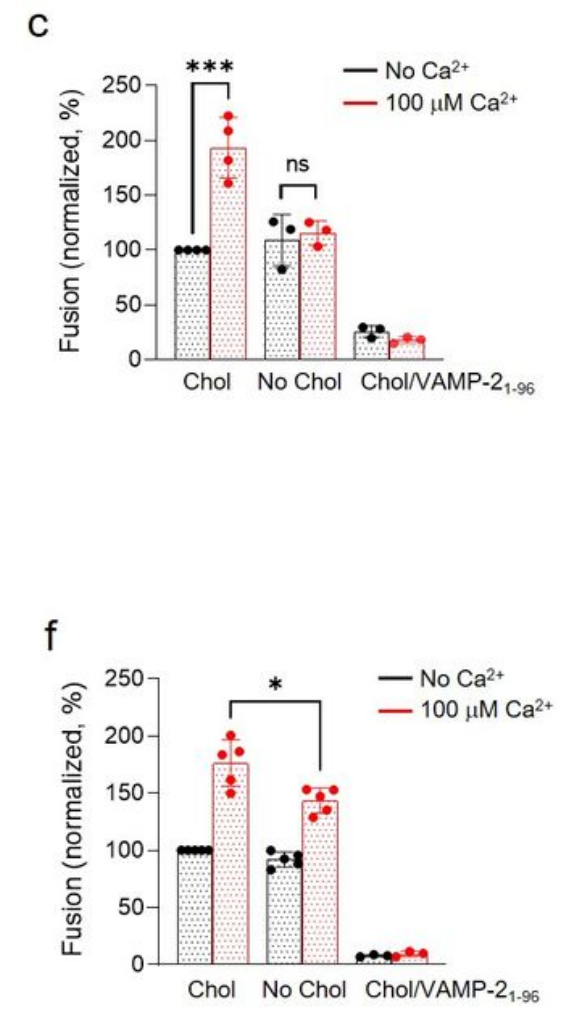

\section{Figure 3}

Cholesterol is not required for liposome-liposome fusion. (a-c) Synaptic vesicle (SV) fusion with the PMliposomes that contain either $25 \%(\mathbf{a}, \mathbf{c})$ or $0 \%(b, c)$ Chol. Only endogenous VAMP-2 and synaptotagmin-1 of native SVs are shown and full fusion is presented for clarity. (d-f) Instead of native LDCVs or SVs, Vliposomes that incorporated the full-length synaptotagmin-1 and VAMP-2 were incubated with PMliposomes that contained either 25\% (d) or 0\% (e) Chol. Both V-liposomes and PM-liposomes are LUVs. Lipid composition of the PM-liposomes is described in Figure 2. Lipid composition of V-liposomes: 55\% PC, $20 \%$ PE, 15\% PS, and 10\% Chol. (c,f) Fusion is presented as a percentage of basal fusion with Cholcontaining PM-liposomes in the absence of $\mathrm{Ca}^{2+}$. Data in $\mathbf{c}$ and $\mathbf{f}$ are mean $\pm \mathrm{SD}$ from $3 \sim 5$ independent experiments $(\mathrm{n}=3 \sim 5) .{ }^{*}, p<0.05 . * \star \star, p<0.0005$. 
Figure 4

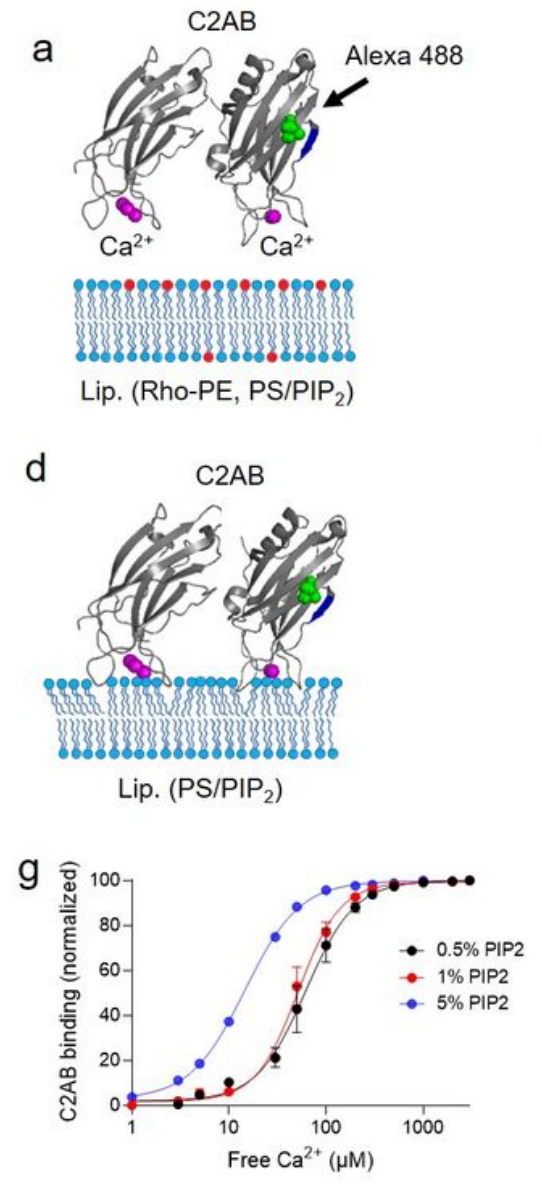

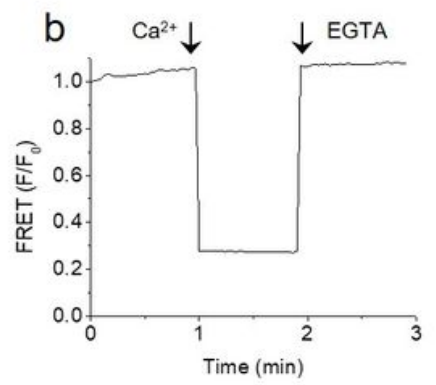
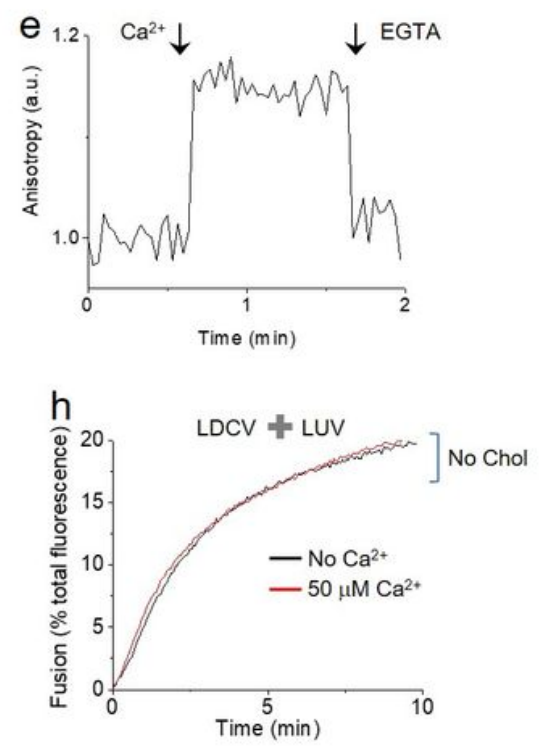

C

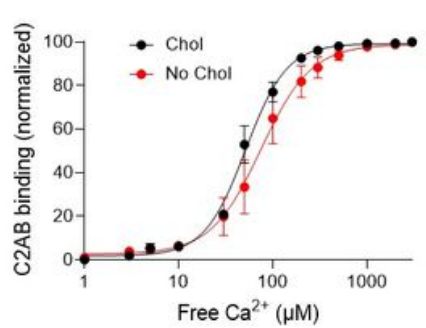

f
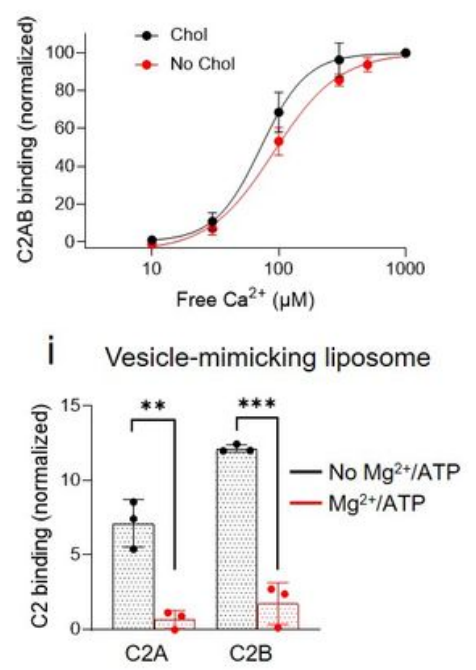

Figure 4

Membrane binding of the C2AB domain of synaptotagmin-1 in the presence or absence of cholesterol. (a-

c) Membrane binding of the $C 2 A B$ domain of synaptotagmin-1 was monitored using FRET in which the

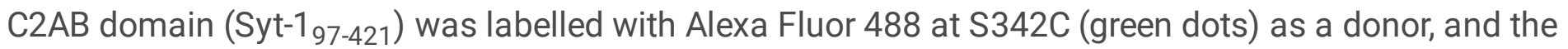
PM-liposomes (Lip.) were labelled with Rhodamine (Rho)-PE (red) as an acceptor (Online Methods). The PM-liposomes (protein-free) contained either $25 \%$ (b) or $0 \%$ Chol. (c) C2AB binding is presented as a percentage of maximum $C 2 A B$ binding induced by $1 \mathrm{mM}^{\text {free }} \mathrm{Ca}^{2+}$. Data are mean $\pm \mathrm{SD}$ from 3 5 independent experiments. (d-f) Binding of the C2AB domain to the PM-liposomes (protein-free; lipid composition as in a-c without Rho-PE) was monitored using fluorescence anisotropy. (f) Dose-response curve of $\mathrm{Ca}^{2+}$-dependent $\mathrm{C} 2 \mathrm{AB}$ binding to the PM-liposomes that contain either $25 \%$ or $0 \%$ Chol. Data are mean \pm SD ( $n=3 \sim 4$ independent experiments). (g) FRET was conducted to monitor C2AB binding to liposomes as in Fig. 4a-c. PIP 2 was incorporated in the PM-liposomes (25\% cholesterol included, proteinfree). (g) High $\mathrm{PIP}_{2}$ concentration increased $\mathrm{Ca}^{2+}$ sensitivity for $\mathrm{C} 2 \mathrm{AB}$ binding to membranes. Data are mean $\pm S D$ ( $n=3 \sim 5$ independent experiments). (h) LDCV fusion with the PM-liposomes that contain 5\% $\mathrm{PIP}_{2}$ concentration and no Chol. (i) $\mathrm{C} 2 \mathrm{AB}$ binding to V-liposomes was monitored using a tryptophandansyl FRET pair. Neither the C2A nor C2B domain binds to V-liposomes (no PIP 2 ) by $1 \mathrm{mM} \mathrm{Ca}^{2+}$ in the presence of $1 \mathrm{mM} \mathrm{MgCl} / 3 \mathrm{mM}$ ATP. Data are mean $\pm \mathrm{SD}$ ( $\mathrm{n}=3$ independent experiments). $* *, p<0.01$. $\star \star \star, p<0.0005$. 
Figure 5

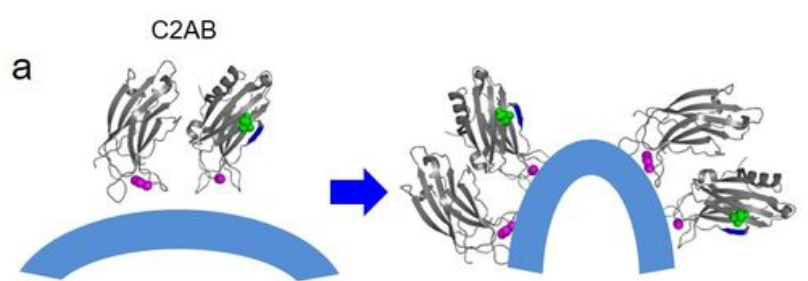

$\operatorname{LUV}(110 \mathrm{~nm})$
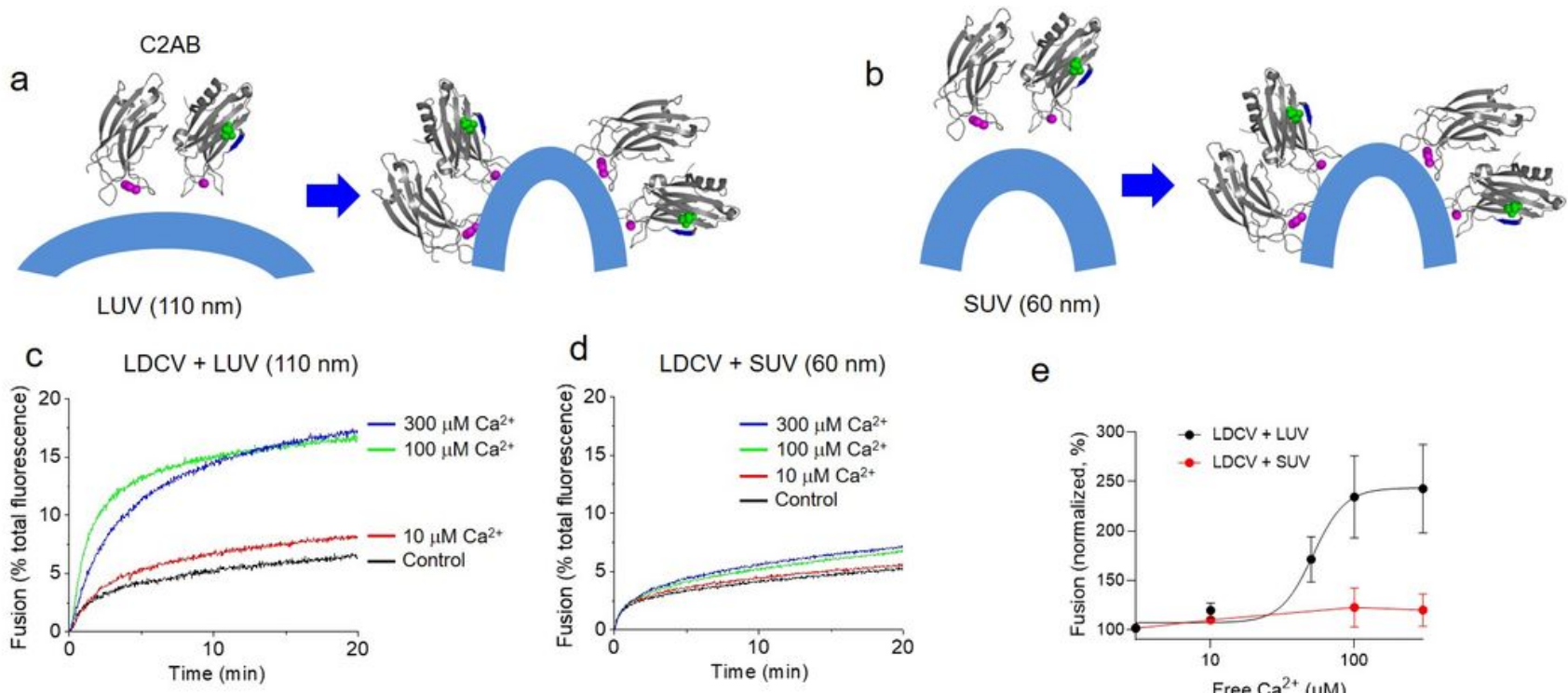

$\operatorname{SUV}(60 \mathrm{~nm})$
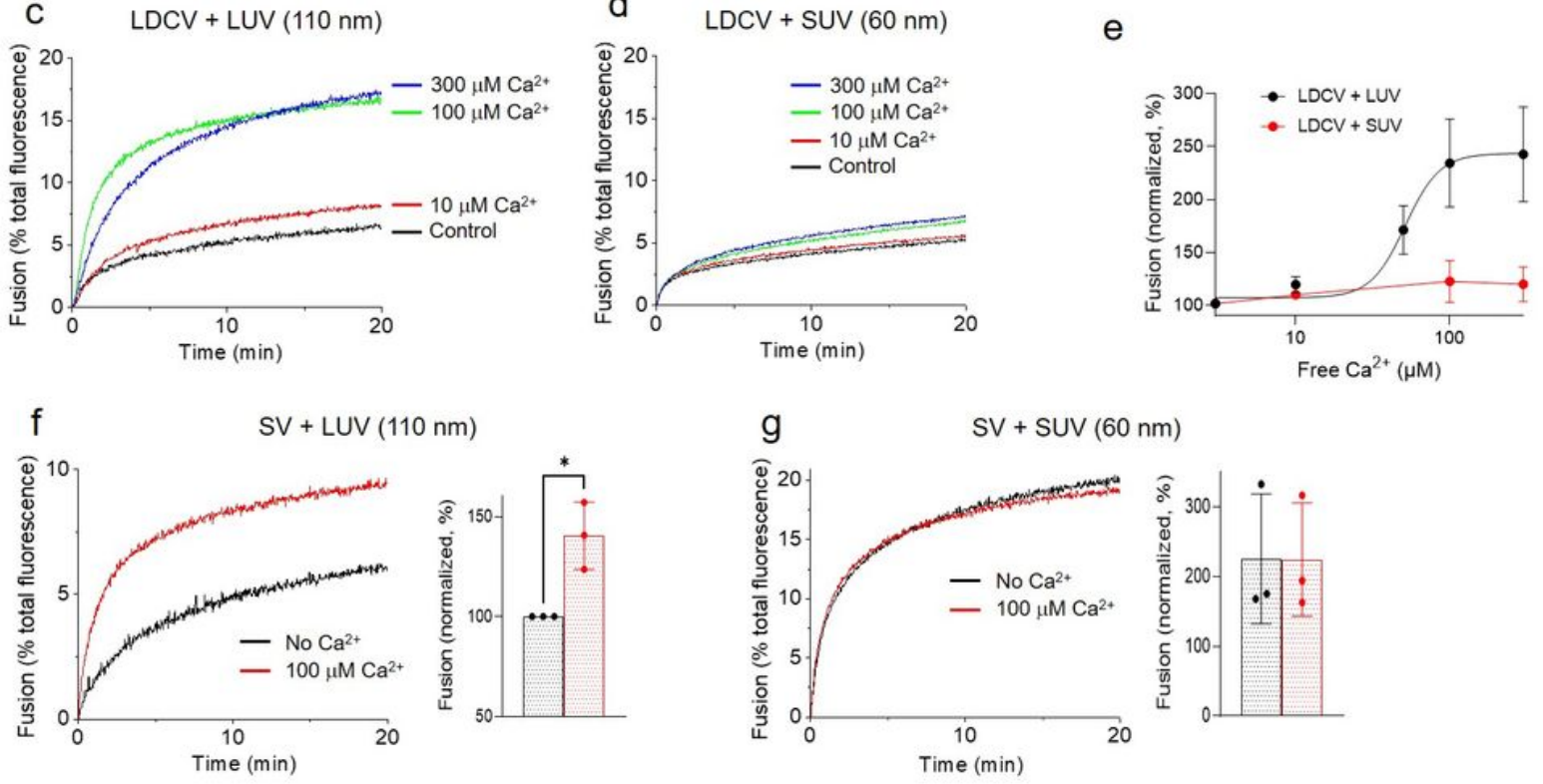

\section{Figure 5}

High curvature strain of membrane increases basal fusion but decreases $\mathrm{Ca}^{2+}$-triggered fusion. $(\mathrm{a}, \mathrm{b})$ Schematic diagram showing membrane bending by membrane binding of the C2AB domain. Large unilamellar vesicles (LUVs), $110 \mathrm{~nm}$ in diameter; small unilamellar vesicles (SUVs), $60 \mathrm{~nm}$ in diameter.

The SUV is already highly curved. (c,d) $\mathrm{Ca}^{2+}$-dependent LDCV fusion with the PM-liposomes, either LUV or SUV. (e) Dose-response curve of $\mathrm{Ca}^{2+}$ on LDCV fusion with either LUV or SUV. $(\mathbf{f}, \mathbf{g})$ SV fusion with the PMliposomes, either LUV or SUV. Mouse SVs are $\sim 45 \mathrm{~nm}$ in diameter. Data in $\mathbf{e , f}, \mathbf{g}$ are mean \pm SD $(\mathrm{n}=3 \sim 7$ independent experiments). ${ }^{*}, p<0.05$. 


\section{Figure 6}
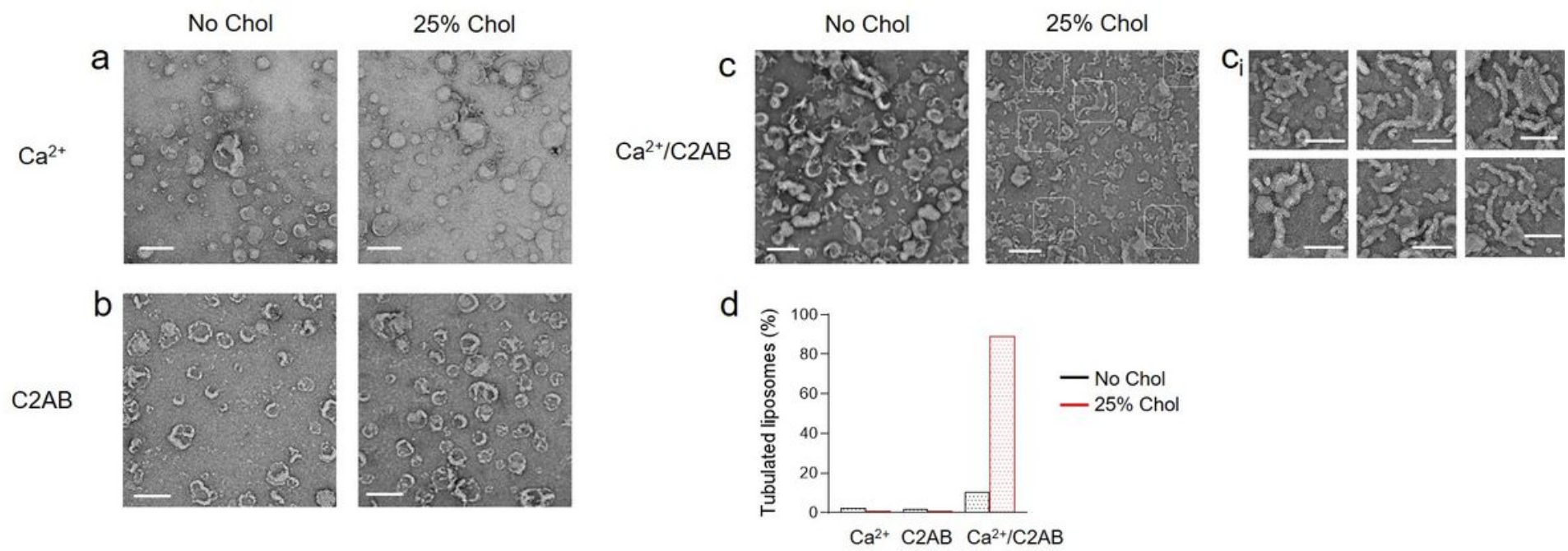

\section{Figure 6}

Cholesterol stabilizes and strengthens membrane deformation induced by the C2AB domain. TEM images of the PM-liposomes in the absence or presence of $25 \%$ Chol, incubated with either $100 \mu \mathrm{M} \mathrm{Ca}^{2+}$ (a), $2 \mu \mathrm{M}$ C2AB domain (b), or $\mathrm{Ca}^{2+} / \mathrm{C} 2 \mathrm{AB}$ domain (c). Scale, $200 \mathrm{~nm}$. ( $\mathbf{c}_{\mathrm{i}}$ ) High magnification images of tubulated liposomes (white dotted lines in the right panel of c). Scale, $100 \mathrm{~nm}$. (d) Quantification of tubulated liposomes ( $n=110 \sim 475$ liposomes in each condition from three independent experiments). 
Figure 7

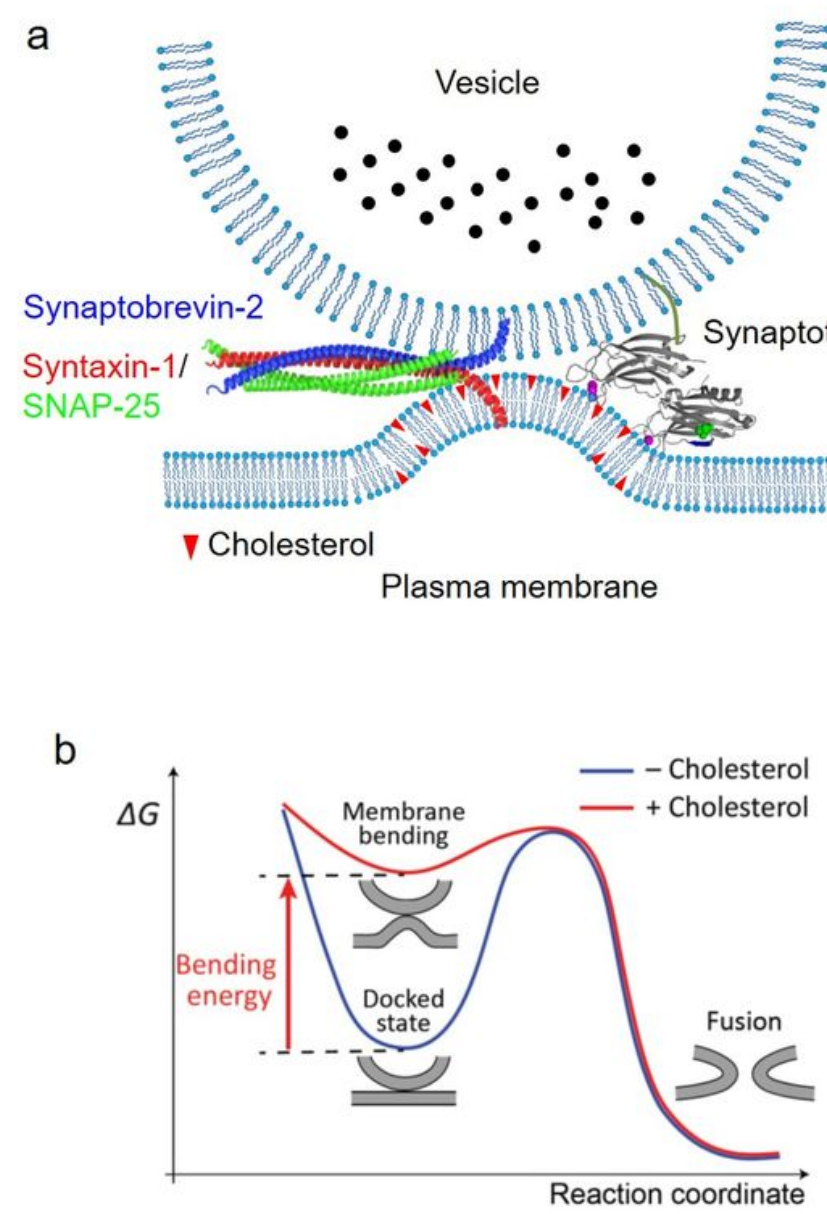

\section{Figure 7}

A schematic diagram summarizing synaptotagmin-1-induced membrane bending for $\mathrm{Ca}^{2+}$-dependent vesicle fusion. (a) Cholesterol stabilizes the plasma membrane deformation and bending caused by synaptotagmin-1. (b) Energy landscapes of vesicle fusion by synaptotagmin-1. Synaptotagmin-1-induced membrane bending enhanced by cholesterol generates the significant membrane bending energy that can drive the membrane fusion by lowering the energy barriers to promote the fusion intermediate.

\section{Supplementary Files}

This is a list of supplementary files associated with this preprint. Click to download.

- Supplementaryfigures.pdf 